\title{
Bispinor Auxiliary Fields in Duality-Invariant Electrodynamics Revisited
}

\author{
E.A. Ivanov, B.M. Zupnik \\ Bogoliubov Laboratory of Theoretical Physics, JINR, \\ 141980 Dubna, Moscow Region, Russia \\ eivanov@theor.jinr.ru, zupnik@theor.jinr.ru
}

\begin{abstract}
Motivated by a recent progress in studying the duality-symmetric models of nonlinear electrodynamics, we revert to the auxiliary tensorial (bispinor) field formulation of the $O(2)$ duality proposed by us in hep-th/0110074, 0303192. In this approach, the entire information about the given duality-symmetric system is encoded in the $O(2)$-invariant interaction Lagrangian which is a function of the auxiliary fields $V_{\alpha \beta}, \bar{V}_{\dot{\alpha} \dot{\beta}}$. We extend this setting to duality-symmetric systems with higher derivatives and show that the recently employed "nonlinear twisted self-duality constraints" amount to the equations of motion for the auxiliary tensorial fields in our approach. Some other related issues are briefly discussed and a few instructive examples are explicitly worked out.
\end{abstract}

PACS: 11.15.-q, 03.50.-z, 03.50.De

Keywords: Electrodynamics, duality, auxiliary fields 


\section{Introduction}

A wide class of the nonlinear electrodynamics models including the renowned Born-Infeld (BI) theory exhibits the on-shell $U(N)$ duality invariance (or a less restrictive discrete self-duality) [1]-[14]. Recently, there was a rebirth of interest in the duality-invariant theories, including those with higher derivatives (see, e.g., [15]-[20]), in connection with the problems of the extended supergravity counterterms. There is the hope that the duality symmetry considerations at the quantum level could play the decisive role in checking the ambitious hypothesis of ultraviolet finiteness of $\mathcal{N}=8,4 D$ supergravity (and/or its higher-dimensional cousins and $\mathcal{N}<8,4 D$ descendants) [15, 16, 17].

About decade ago, we proposed a new general formulation of the duality-invariant theories exploiting the tensorial (bispinor) auxiliary fields [21, 22, 23]. In this formulation, the $U(N)$ or discrete duality symmetries acquire a nice interpretation as the linear off-shell symmetries of the nonlinear interaction constructed out of the auxiliary fields. These symmetries are broken in the total action, since its free bilinear part is not invariant. The famous Noether-Gaillard-Zumino (NGZ) constraint [2, 3] is linearized in this formalism and, as a consequence, the duality symmetry between the Bianchi identities and equations of motion becomes manifest. The basic algebraic equations of motion relate the auxiliary fields to the Maxwell field strengths and, in general, can be solved by some recursive procedure. Substituting the solutions for the auxiliary fields back into the original action, we obtain the perturbative expansion of the nonlinear Lagrangians in terms of the electromagnetic field strengths. In some particular cases of the $U(1)$ duality invariant models, the basic equations are reduced to the algebraic equations of the orders 2, 3 or 4, and it becomes possible to write their solutions (and the eventual actions) in a closed form. In particular, the quadratic equation gives rise to the notorious Born-Infeld (BI) theory.

One of the aims of the present paper is to argue that the methods recently proposed in [17, 18, 19] for the systematic construction of various duality-invariant nonlinear Lagrangians are in fact equivalent to our approach [21, 22, 23. In particular, "the nonlinear twisted duality constraint" of [17, 18, 19] is none other than the equation of motion for our bispinor auxiliary field. Realizing this motivated us to return to the original formulation in order to see how the latest developments in the duality stuff can be inscribed into its framework.

In Section 2 we review our auxiliary-field formalism for the $U(1)$ (or $O(2)$ ) duality symmetric electrodynamics models in the simplest case without derivatives of the field strengths. We demonstrate that the model with the simplest quartic auxiliary $O(2)$ invariant interaction $\frac{1}{2} V^{2} \bar{V}^{2}$ [21] just amounts to the duality-invariant model recently discussed, in parallel with the BI theory, in [17, 18]. In our method this model corresponds to the auxiliary algebraic equation of the fifth order. We discuss the Legendre-type transformation of the scalar variable $\nu=V^{2}$ to the $\mu$ representation [23], which simplifies both solving the auxiliary equations and constructing the Lagrangians of the dualityinvariant systems in the standard representation through the Maxwell field strengths. We also consider the combined $(F, V, \mu)$ representation of these Lagrangians, with both the bispinor and the scalar auxiliary fields. The $\mu$ or $V$ representations arise after elimination 
of the appropriate auxiliary field.

The authors of [17, 19] studied the duality-invariant theories with higher derivatives. In Section 3 we discuss how to treat such theories in the framework of our auxiliary-field formulation. Once again, the basic requirement is the $O(2)$ invariance of the interaction which is now constructed out of the auxiliary fields and their derivatives. Though the equation for the auxiliary fields contains derivatives, it still can be solved recursively, giving rise to various Lagrangians with higher derivatives of the field-strengths $F_{\alpha \beta}, F_{\dot{\alpha} \dot{\beta}}$, such that the corresponding theories are guaranteed to exhibit duality invariance, with the relevant generalized NGZ condition being satisfied.

In Appendix A we present the precise correspondence between the vector notation used in [17, 18, 19] and our complex spinor notation.

In Appendix B we show that the Schrödinger nonlinear constraints [24] (pertinent to the Born-Infeld theory as a particular case of duality-symmetric systems) are readily reproduced in our approach.

The Lorentz non-covariant but manifestly $O(2)$ duality invariant version of our Lagrangian with auxiliary fields is discussed in Appendix C.

In the accompanying paper [25] we will analyze, in more detail compared to [22], the nonabelian $U(N)$ duality within our tensorial auxiliary field formulation. In the near future we also plan to extend this formulation by incorporating scalar coset fields which are necessary ingredients of the duality-symmetric supergravities.

\section{Auxiliary variables in nonlinear electrodynamics}

\subsection{The standard duality setting}

We use the $S L(2, C)$ spinor representation for the electromagnetic gauge field $A_{\alpha \dot{\beta}}$ and the complex dimensionless Maxwell field strengths $F_{\alpha \beta}$ and $\bar{F}_{\dot{\alpha} \dot{\beta}}$. The general nonlinear Lagrangian with no derivatives on the field strengths is constructed from the scalar quantities $\varphi=F^{\alpha \beta} F_{\alpha \beta}=(F F)$ and $\bar{\varphi}=(\bar{F} \bar{F})$

$$
L(\varphi, \bar{\varphi})=-\frac{1}{2}(\varphi+\bar{\varphi})+L^{i n t}(\varphi, \bar{\varphi}) .
$$

The physical rescaling of this dimensionless Lagrangian includes the coupling constant $f$ of dimension $[f]=-2$ and the field strengths $F, \bar{F}$ of the standard dimension

$$
L\left(F^{2}, \bar{F}^{2}\right) \rightarrow \frac{1}{f^{2}} L\left(f^{2} F^{2}, f^{2} \bar{F}^{2}\right) .
$$

In the more familiar tensor notation (see Appendix A) the Lagrangian $L(\varphi, \bar{\varphi})$ is rewritten in terms of the standard Maxwell field strength $F_{m n}$ and its dual $\tilde{F}_{m n}$ as $L(\varphi, \bar{\varphi})=\tilde{L}(t, z)$, where $\varphi=t+i z$ and $t=\frac{1}{4} F_{m n} F^{m n}, z=\frac{1}{4} \tilde{F}_{m n} F^{m n}$.

The nonlinear equations of motion following from (2.1) and the Bianchi identities for the field strengths have the form

$$
\begin{aligned}
& E_{\alpha \dot{\alpha}}:=\partial_{\alpha}^{\dot{\beta}} \bar{P}_{\dot{\alpha} \dot{\beta}}(F)-\partial_{\dot{\alpha}}^{\beta} P_{\alpha \beta}(F)=0, \\
& B_{\alpha \dot{\alpha}}:=\partial_{\alpha}^{\dot{\beta}} \bar{F}_{\dot{\alpha} \dot{\beta}}-\partial_{\dot{\alpha}}^{\beta} F_{\alpha \beta}=0 .
\end{aligned}
$$


Here, the nonlinear bispinor fields

$$
P_{\alpha \beta}=i \frac{\partial L}{\partial F^{\alpha \beta}}, \quad \bar{P}_{\dot{\alpha} \dot{\beta}}=\overline{P_{\alpha \beta}}=-i \frac{\partial L}{\partial \bar{F}^{\dot{\alpha} \dot{\beta}}}
$$

correspond to the standard self-dual and anti-self-dual fields $G_{m n}^{ \pm}$used in the tensor notation (see (A.12) ).

The set of equations (2.3) and (2.4) is covariant under the $O(2)$ duality transformations

$$
\delta_{\omega} F_{\alpha \beta}=\omega P_{\alpha \beta}, \quad \delta_{\omega} P_{\alpha \beta}=-\omega F_{\alpha \beta},
$$

provided that the nonlinear $O(2)$ duality NGZ condition [2, 3] for the Lagrangian $L$ is satisfied

$$
F_{\alpha \beta} F^{\alpha \beta}+P_{\alpha \beta} P^{\alpha \beta}-\text { c.c }=0 \quad \Longleftrightarrow \quad \varphi-\bar{\varphi}-4\left[\varphi\left(L_{\varphi}\right)^{2}-\bar{\varphi}\left(L_{\bar{\varphi}}\right)^{2}\right]=0 .
$$

This condition (which is $O(2)$ invariant by itself) guarantees the consistency of the $O(2)$ transformations (2.6) with the definition (2.5).

In the on-shell equations (2.3), (2.4) and the $O(2)$ transformations (2.6), the field strength $F_{\alpha \beta}$ and its conjugate are treated as independent complex variables. Off shell the field strengths are expressed in terms of the gauge potential $A_{m}$. It is impossible to implement the $O(2)$ transformations off shell on the single real vector field $A_{m}$, which implies that the total off-shell action cannot possess $O(2)$ duality invariance.

Still for the Lagrangians of the duality-invariant systems there exists the partially $O(2)$ invariant Gaillard-Zumino (GZ) representation [2]

$$
L=\frac{i}{2}(\bar{P} \bar{F}-P F)+I(\varphi, \bar{\varphi})=\frac{1}{4} \tilde{G}^{m n} F_{m n}+\tilde{I}(t, z) .
$$

Here $I$ (or $\tilde{I}$ ) are some $O(2)$ invariants. This representation can be easily proved by varying the general Lagrangian (2.1) with respect to the transformations (2.6) with taking into account the NGZ condition (2.7), which gives

$$
\delta L=i \omega(\varphi-\bar{\varphi}) \text {. }
$$

This coincides with the variation of the bilinear term in (2.8); i.e. the remaining term $I$ is indeed duality-invariant.

The representation (2.8) on its own is not too useful since no systematic way to construct the invariants $I$ or $\tilde{I}$ was known 1 . Such a way is suggested by the formulation with the auxiliary tensorial fields.

\subsection{Reformulation through tensorial auxiliary fields}

The basic $(F, V)$-representation of the Lagrangian of general duality-invariant system in our formalism [21, 23] involves the bispinor field-strengthes $F_{\alpha \beta}$ and $\bar{F}_{\dot{\alpha} \dot{\beta}}$ and the auxiliary

\footnotetext{
${ }^{1}$ This problem actually amounts to solving the nonlinear NGZ condition (2.7).
} 
fields $V_{\alpha \beta}$ and $\bar{V}_{\dot{\alpha} \dot{\beta}}$

$$
\begin{aligned}
& \mathcal{L}(V, F)=\mathcal{L}_{2}(V, F)+E(\nu, \bar{\nu}) \\
& \mathcal{L}_{2}(V, F)=\frac{1}{2}\left(F^{2}+\bar{F}^{2}\right)+V^{2}+\bar{V}^{2}-2(V \cdot F+\bar{V} \cdot \bar{F}) .
\end{aligned}
$$

Here $\mathcal{L}_{2}(V, F)$ is the bilinear part through which the Maxwell field strength enters the action and $E(\nu, \bar{\nu})$ is the nonlinear interaction involving only auxiliary fields. We use the convenient scalar variables

$$
\nu=V^{2}, \quad \bar{\nu}=\bar{V}^{2}
$$

The $O(2)$ transformations of the involved fields read

$$
\begin{aligned}
& \delta_{\omega} V_{\alpha \beta}=-i \omega V_{\alpha \beta}, \quad \delta_{\omega} \bar{V}_{\dot{\alpha} \dot{\beta}}=i \omega \bar{V}_{\dot{\alpha} \dot{\beta}}, \quad \delta_{\omega} \nu=-2 i \omega \nu, \\
& \delta_{\omega} F_{\alpha \beta}=i \omega\left(F_{\alpha \beta}-2 V_{\alpha \beta}\right), \quad \delta_{\omega} \bar{F}_{\dot{\alpha} \dot{\beta}}=-i \omega\left(\bar{F}_{\dot{\alpha} \dot{\beta}}-2 \bar{V}_{\dot{\alpha} \dot{\beta}}\right) .
\end{aligned}
$$

The Bianchi equation (2.4) together with the dynamical equation of motion corresponding to the Lagrangian (2.10),

$$
\begin{aligned}
& \partial_{\alpha}^{\dot{\beta}} \bar{P}_{\dot{\alpha} \dot{\beta}}(V, F)-\partial_{\dot{\alpha}}^{\beta} P_{\alpha \beta}(V, F)=0, \\
& P_{\alpha \beta}(F, V)=i \frac{\partial \mathcal{L}(V, F)}{\partial F^{\alpha \beta}}=i\left(F_{\alpha \beta}-2 V_{\alpha \beta}\right), \quad \bar{P}_{\dot{\alpha} \dot{\beta}}(F, V)=\overline{\left(P_{\alpha \beta}(F, V)\right)},
\end{aligned}
$$

constitute the set of equations manifestly covariant under these $O(2)$ transformations. The latter take the more familiar equivalent form just in terms of the variables $P_{\alpha \beta}, F_{\alpha \beta}$ (c.f. $(2.6))$ :

$$
\delta_{\omega} P_{\alpha \beta}(F, V)=-\omega F_{\alpha \beta}, \quad \delta_{\omega} F_{\alpha \beta}=\omega P_{\alpha \beta}(F, V) .
$$

The full set of the equations of motion associated with the Lagrangian (2.11), including the algebraic ones for the auxiliary fields $V_{\alpha \beta}, \bar{V}_{\dot{\alpha} \dot{\beta}}$, is duality-covariant only under a certain restriction on the interaction part $E(\nu, \bar{\nu})$. This restriction actually amounts to the constraint (2.7), but looks much simpler and has the obvious group-theoretical meaning.

The algebraic equations for $V_{\alpha \beta}, \bar{V}_{\dot{\alpha} \dot{\beta}}$ are the basic equations of our formalism. They arise from varying $\mathcal{L}(V, F)$ with respect to $V_{\alpha \beta}, \bar{V}_{\dot{\alpha} \dot{\beta}}$ and have the form

$$
F_{\alpha \beta}=V_{\alpha \beta}+\frac{1}{2} \frac{\partial E}{\partial V^{\alpha \beta}}=V_{\alpha \beta}\left(1+E_{\nu}\right) \quad \text { and c.c. } .
$$

Their important corollaries are, in particular,

$$
\text { (a) } V \cdot F=\nu\left(1+E_{\nu}\right), \quad \text { (b) } \varphi=\nu\left(1+E_{\nu}\right)^{2} \text {. }
$$

The perturbative solution of (2.17) for functions $G$ or $L_{\varphi}$,

$$
\begin{aligned}
& V_{\alpha \beta}(F)=F_{\alpha \beta} G(\varphi, \bar{\varphi}), \\
& G(\varphi, \bar{\varphi})=\frac{1}{2}-L_{\varphi}=\left(1+E_{\nu}\right)^{-1}
\end{aligned}
$$


allows us to construct the nonlinear Lagrangian $L(\varphi, \bar{\varphi})$ from the Lagrangian $\mathcal{L}(F, V(F))$. Using the relations (2.18b) and (2.20), one can also express $\nu$ and $E_{\nu}$ through $\varphi$ and $L_{\varphi}$ :

$$
E_{\nu}=\frac{1+2 L_{\varphi}}{1-2 L_{\varphi}}, \quad \nu=\frac{1}{4} \varphi\left(1-2 L_{\varphi}\right)^{2} .
$$

In this way, any $L(\varphi, \bar{\varphi})$ (non-singular in the weak-field limit) can be restored by the appropriate function $E(\nu, \bar{\nu})$. The duality-invariant systems correspond to the special choice of $E(\nu, \bar{\nu})$. To find out the appropriate restriction on this function, we need to rewrite the NGZ condition (2.7) in our formalism.

Using the definition (2.15) together with the relations (2.18) and (2.21), it is easy to find

$$
F^{2}+P^{2}=\varphi\left(1-4 L_{\varphi}^{2}\right)=4 \nu E_{\nu} .
$$

Then the nonlinear NGZ condition (2.7) is reduced to the linear constraint:

$$
F^{2}+P^{2}-\bar{F}^{2}-\bar{P}^{2}=0 \quad \Longleftrightarrow \quad \nu E_{\nu}-\bar{\nu} E_{\bar{\nu}}=0
$$

This is none other than the condition of invariance of the interaction $E(\nu, \bar{\nu})$ under the $O(2)$ transformations (2.12):

$$
\delta_{\omega} E=2 i \omega\left(\bar{\nu} E_{\bar{\nu}}-\nu E_{\nu}\right)=0 .
$$

The general solution of this condition is obviously

$$
E(\nu, \bar{\nu})=\mathcal{E}(a), \quad a=\nu \bar{\nu}=V^{2} \bar{V}^{2}
$$

Now, taking into account (2.23), one can check the $O(2)$ covariance of the basic algebraic equation (2.17) for this particular subclass of the self-interactions $E(\nu, \bar{\nu})$. In fact, one can reverse the argument and directly deduce (2.23) as the necessary and sufficient condition of covariance of Eq. (2.17) under the transformations (2.12), (2.13). For what follows, it will be instructive to rewrite (2.17) and its conjugate in the form specialized to the duality-covariant case

$$
F_{\alpha \beta}-V_{\alpha \beta}=V_{\alpha \beta} \bar{V}^{2} \mathcal{E}_{a}(a), \quad \bar{F}_{\dot{\alpha} \dot{\beta}}-\bar{V}_{\dot{\alpha} \dot{\beta}}=\bar{V}_{\dot{\alpha} \dot{\beta}} V^{2} \mathcal{E}_{a}(a)
$$

It is now straightforward to reveal how the general GZ representation (2.8) for the Lagrangians of the duality-invariant systems looks in our approach. We rewrite the offshell action (2.10) in the form

$$
\mathcal{L}(F, V)=\frac{i}{2}[\bar{P}(F, V) \bar{F}-P(F, V) F]+\left[V^{2}-(F V)\right]+\left[\bar{V}^{2}-(\bar{F} \bar{V})\right]+\mathcal{E}(\nu \bar{\nu})
$$

and observe that the $(V, F)$ analog of the $O(2)$ invariant function $I(\varphi, \bar{\varphi})$ defined in (2.8) is given by

$$
I(V, F)=\left[V^{2}-(F V)\right]+\left[\bar{V}^{2}-(\bar{F} \bar{V})\right]+\mathcal{E}(\nu \bar{\nu})
$$


It is manifestly invariant under the duality rotations (2.12), (2.13). Expressing $V_{\alpha \beta}, \bar{V}_{\dot{\alpha} \dot{\beta}}$ in it through $F_{\alpha \beta}, \bar{F}_{\dot{\alpha} \dot{\beta}}$ by Eqs. (2.17), we obtain the general representation for $I(\varphi, \bar{\varphi})$ :

$$
I(\varphi, \bar{\varphi})=-2 a \mathcal{E}_{a}(a)+\mathcal{E}(a), \quad a=\nu(\varphi, \bar{\varphi}) \bar{\nu}(\bar{\varphi}, \varphi) .
$$

The basic (and most difficult) problem of the approach discussed consists in expressing, for the given $\mathcal{E}(a)$, the variable $a$ through the original variables $\varphi$ and $\bar{\varphi}$. From the auxiliary field equation (2.18 a) (and its conjugate) one can derive the real algebraic equation [23]

$$
\left(1+a \mathcal{E}_{a}^{2}\right)^{2} \varphi \bar{\varphi}=a\left[(\varphi+\bar{\varphi}) \mathcal{E}_{a}+\left(1-a \mathcal{E}_{a}^{2}\right)^{2}\right]^{2},
$$

which can be used for this purpose. The general expression for the Lagrangian (2.10) in terms of $\varphi$ and $\bar{\varphi}$, after the repeated use of the equations (2.17) and (2.18), is obtained as follows

$$
L^{s d}(\varphi, \bar{\varphi})=\mathcal{L}(V(F), F)=-\frac{1}{2} \frac{(\varphi+\bar{\varphi})\left(1-a \mathcal{E}_{a}^{2}\right)+8 a^{2} \mathcal{E}_{a}^{3}}{1+a \mathcal{E}_{a}^{2}}+\mathcal{E}(a),
$$

where $a$ is related to $\varphi, \bar{\varphi}$ by Eq. (2.30).

To summarize, all $O(2)$ duality-symmetric systems of nonlinear electrodynamics without derivatives on the field strengths are parametrized by the $O(2)$ invariant off-shell interaction of the auxiliary fields $\mathcal{E}(a)$ which is a function of the real quartic combination of the auxiliary fields. This universality seems to be the basic advantage of the approach with tensorial auxiliary fields. The problem of constructing $O(2)$ duality-symmetric systems is reduced to choosing (at will) one or another specific $\mathcal{E}(a)$.

After passing to the tensorial notation (see Appendix A), our basic auxiliary field equation (2.26) (proposed more than ten years ago) is surprisingly recognized as the "nonlinear twisted self-duality constraint" of Refs. [17, 18, 19], while the $O(2)$ invariant function $\mathcal{E}(\nu \bar{\nu})$ as the "duality-invariant source of deformation". It should be pointed out that in our approach this constraint is by no means postulated but naturally arises as the algebraic equations of motion associated with the new off-shell universal Lagrangian (2.10) for the duality-symmetric systems, in which $E(\nu, \bar{\nu})=\mathcal{E}(\nu \bar{\nu})$. The auxiliary tensorial (or bispinor) fields $V_{\alpha \beta}, \bar{V}_{\dot{\alpha} \dot{\beta}}$ appearing in (2.10) are entirely unconstrained off shell: there is no need to express them, e.g., through the second gauge field or to subject them to any other conditions. The final nonlinear self-dual Lagrangian as a function of the Maxwell field strengths $F_{\alpha \beta}, \bar{F}_{\dot{\alpha} \dot{\beta}}$ comes out as the result of eliminating the tensorial auxiliary fields by their equations of motion.

It is worthwhile here to recall that in [2, 3, 8, 9] there was developed another approach to solving the NGZ constraint (2.7) and restoring the appropriate Lagrangian $L(\varphi, \bar{\varphi})$, based on reducing (2.7) to the Courant-Gilbert nonlinear differential equation. It was found that the whole family of the duality-invariant Lagrangians is parametrized by some real function of one argument. Despite this formal resemblance, there are essential distinctions between this construction and our procedure. In contrast to the former, our approach guarantees the analyticity of $L(\varphi, \bar{\varphi}) 2$. Also, at all steps we make use of the algebraic equations, while the approach of [2, 3, 8, 9] exploits the differential equations.

\footnotetext{
${ }^{2}$ The perturbative linear relation of the general self-dual Lagrangian with the corresponding real function of $(\varphi \bar{\varphi})$ was analyzed in [4].
} 
Finally, we notice that, besides the continuous $O(2)$ duality, some models of nonlinear electrodynamics reveal the so called "discrete duality" or "duality by Legendre transformation" [2]. It also has a simple realization in terms of the auxiliary fields [22]:

$$
V \rightarrow-i V, \quad \nu \rightarrow-\nu, \quad \bar{V} \rightarrow i \bar{V}, \quad \bar{\nu} \rightarrow-\bar{\nu}, \quad E(\nu, \bar{\nu})=E(-\nu,-\bar{\nu}) .
$$

\subsection{Alternative auxiliary field representations}

The representation (2.31) together with the algebraic equation (2.30) in principle solve the problem of finding the explicit form of the action of $O(2)$ duality invariant system by a fixed $\mathcal{E}(a)$ in terms of the Maxwell strengths (at least, as a power series in $\varphi$ and $\bar{\varphi}$ ). However, in practice it is frequently more convenient to deal with the set of the auxiliary complex variables $\mu$ and $\bar{\mu}$ instead of the original ones $\nu$ and $\bar{\nu}$. These two sets of variables are related to each other through the Legendre transformation

$$
\begin{aligned}
& \mu:=E_{\nu}, \quad \bar{\mu}:=E_{\bar{\nu}}, \quad E-\nu E_{\nu}-\bar{\nu} E_{\bar{\nu}}:=H(\mu, \bar{\mu}), \\
& \nu=-H_{\mu}, \quad \bar{\nu}=-H_{\bar{\mu}} \quad E=H-\mu H_{\mu}-\bar{\mu} H_{\bar{\mu}} .
\end{aligned}
$$

The function $H(\mu, \bar{\mu})$ provides an alternative representation of the nontrivial interaction $E \neq 0$. Under the off-shell $O(2)$ duality the variables $\mu$ and $\bar{\mu}$ are evidently transformed as

$$
\delta_{\omega} \mu=2 i \omega \mu, \quad \delta_{\omega} \bar{\mu}=-2 i \omega \bar{\mu},
$$

so the $O(2)$ duality invariance requires

$$
H(\mu, \bar{\mu})=I(b), \quad b=\mu \bar{\mu} .
$$

From (2.33), (2.34) one finds

$$
\mathcal{E}(a)-2 a \mathcal{E}_{a}(a)=I(b), \quad a=b I_{b}^{2}, \quad b=a \mathcal{E}_{a}^{2}, \quad \mathcal{E}_{a}=-\left(I_{b}\right)^{-1} .
$$

In order to guarantee the $(\mu, \bar{\mu}) \leftrightarrow(\nu, \bar{\nu})$ transform to be invertible, we are led to assume that $\mathcal{E}_{a}(a=0) \neq 0, I_{b}(b=0) \neq 0$, i.e. that $\mathcal{E}(a)$ starts with the term $\propto a$.

The basic algebraic equations of this representation directly follow from Eq. (2.18b)

$$
\varphi=-(1+\mu)^{2} H_{\mu}=-(\bar{\mu}+2 b+b \mu) I_{b} \quad \text { and c.c. . }
$$

They enable expressing $\mu, \bar{\mu}$ in terms of $\varphi, \bar{\varphi}$. The equation for the invariant variable $b$ also directly follows from (2.30), and it reads [23]

$$
(b+1)^{2} \varphi \bar{\varphi}=b\left[\varphi+\bar{\varphi}-I_{b}(b-1)^{2}\right]^{2} .
$$

The relevant expression for the general Lagrangian $\mathcal{L}(V(F), F)$ can be easily obtained from the expression (2.31):

$$
L^{s d}(\varphi, \bar{\varphi})=-\frac{1}{2}\left(\varphi+\bar{\varphi}+4 b I_{b}\right) \frac{1-b}{1+b}+I(b) .
$$


It is worth noting that one can reproduce the algebraic relations (2.37) from the new Lagrangian with $\mu$ as an independent complex auxiliary field:

$$
\tilde{L}(\varphi, \mu)=\frac{\varphi(\mu-1)}{2(\mu+1)}+\frac{\bar{\varphi}(\bar{\mu}-1)}{2(\bar{\mu}+1)}+I(\mu \bar{\mu}) .
$$

Varying (2.40) with respect to $\mu, \bar{\mu}$ yields just (2.37), from which one can derive Eq. (2.38) without any reference to the original $\nu, \bar{\nu}$ representation. Eliminating $\mu$ and $\bar{\mu}$ from (2.40) in terms of $\varphi, \bar{\varphi}$ and $b$ with the help of (2.37) and its corollary (2.38), we recover the Lagrangian (2.39) .

The dual field strength $P_{\alpha \beta}$ appearing in the nonlinear equation of motion for $F_{\alpha \beta}$ derived directly from the extended Lagrangian (2.40) is as follows

$$
P_{\alpha \beta}(F, \mu)=2 i F_{\alpha \beta} \frac{\partial \tilde{L}(\varphi, \mu)}{\partial \varphi}=i F_{\alpha \beta} \frac{\mu-1}{\mu+1} .
$$

The consistency of the nonlinear $O(2)$ transformations,

$$
\delta_{\omega} F_{\alpha \beta}=\omega P_{\alpha \beta}, \quad \delta_{\omega} P_{\alpha \beta}=-\omega F_{\alpha \beta},
$$

can be easily checked, assuming that the auxiliary variables $\mu, \bar{\mu}$ transform as in (2.35).

The Lagrangian (2.40) provides the convenient off-shell description of the general duality-invariant system of nonlinear electrodynamics with an extra complex auxiliary field. The free Lagrangian corresponds to the limit $\mu=0, \tilde{L}(\varphi, 0)=-\frac{1}{2}(\varphi+\bar{\varphi})$.

There also exists a combined representation for the general self-dual Lagrangian, with two auxiliary fields, $V_{\alpha \beta}$ and $\mu$,

$$
\mathcal{L}(F, V, \mu)=\frac{1}{2}\left(F^{2}+\bar{F}^{2}\right)-2(V F)-(\bar{V} \bar{F})+V^{2}(1+\mu)+(1+\bar{\mu}) \bar{V}^{2}+I(\mu \bar{\mu}) .
$$

Eliminating the bispinor auxiliary field via its algebraic equation $V_{\alpha \beta}=F_{\alpha \beta} /(1+\mu)$, we come back to the Lagrangian (2.40). On the other hand, the $(\mu, \bar{\mu})$ equations give us just the relations (2.34) between $\nu=V^{2}, \bar{\nu}=\bar{V}^{2}$ and $\mu, \bar{\mu}$

$$
\nu+\bar{\mu} I_{b}=0, \quad \bar{\nu}+\mu I_{b}=0 .
$$

The equations for $\mu(\nu, \bar{\nu})$ can be solved recursively in the general case and explicitly for some special functions $I_{b}$, thus yielding the original $(F, V)$ representation.

\subsection{Examples}

I. Born-Infeld model. The classical example of the $O(2)$ duality invariant model of nonlinear electrodynamics is the renowned Born-Infeld theory. In our approach it has a more simple description in the $\mu$ (or $b$ ) representation. The corresponding function $I(b)$ is as follows

$$
I^{B I}(b)=\frac{2 b}{b-1}, \quad I_{b}^{B I}=-\frac{2}{(b-1)^{2}} .
$$


The equation (2.38) becomes quadratic in this case:

$$
\varphi \bar{\varphi} b^{2}+\left[2 \varphi \bar{\varphi}-(\varphi+\bar{\varphi}+2)^{2}\right] b+\varphi \bar{\varphi}=0 .
$$

It can be explicitly solved as [23]:

$$
b=\frac{4 \varphi \bar{\varphi}}{[2(1+Q)+\varphi+\bar{\varphi}]^{2}}, \quad Q(\varphi)=\sqrt{1+\varphi+\bar{\varphi}+(1 / 4)(\varphi-\bar{\varphi})^{2}} .
$$

After substituting (2.45), (2.47) into (2.39) we recover the standard Born-Infeld Lagrangian

$$
L^{B I}(\varphi, \bar{\varphi})=1-\sqrt{1+\varphi+\bar{\varphi}+(1 / 4)(\varphi-\bar{\varphi})^{2}} .
$$

The formulation of the BI theory in the original $a$ (or $\nu$ ) representation is also possible but it proves to be much more involved. The original variable $a$ is related to $b$ as

$$
a=\frac{4 b}{(1-b)^{4}}
$$

and for $\mathcal{E}(a)$ one obtains the representation

$$
\mathcal{E}^{B I}(a)=\frac{2 b(a)[1+b(a)]}{[1-b(a)]^{2}}=2\left[2 t^{2}(a)+3 t(a)+1\right]
$$

where $t(a)$ is defined by the 4 -th order equation

$$
t^{4}+t^{3}-(1 / 4) a=0, \quad t(\nu=\varphi=0)=-1 .
$$

Solving it, one can find closed expressions for both $t(a)$ and $\mathcal{E}^{B I}(a)$, but they look not too illuminating. Up to the $3 \mathrm{~d}$ order in $a$ :

$$
\mathcal{E}^{B I}(a)=\frac{1}{2} a-\frac{1}{8} a^{2}+\frac{3}{32} a^{3}+O\left(a^{4}\right) .
$$

II. The simplest interaction (SI) model. This $O(2)$ duality invariant model is one of those considered in [17] and referred to as the BN model in [18]. It corresponds to the following constraint on the Lagrangian $L^{S I}(\varphi, \bar{\varphi})=\tilde{L}^{B N}(t, z)$ [18]:

$$
\left(1+\tilde{L}_{t}^{S I}-i \tilde{L}_{z}^{S I}\right)-\frac{1}{8}(t-i z)\left(1-\tilde{L}_{t}^{S I}-i \tilde{L}_{z}^{S I}\right)^{2}\left(1-\tilde{L}_{t}^{S I}+i \tilde{L}_{z}^{S I}\right)=0 .
$$

It can be rewritten through the variables $\varphi, \bar{\varphi}$ as

$$
\left(1+2 L_{\varphi}^{S I}\right)-\frac{1}{8} \bar{\varphi}\left(1-2 L_{\bar{\varphi}}^{S I}\right)^{2}\left(1-2 L_{\varphi}^{S I}\right)=0 \quad \text { and c.c. },
$$

where we made use of the correspondence

$$
\partial_{\varphi}=\frac{1}{2}\left(\partial_{t}-i \partial_{z}\right), \quad \partial_{\bar{\varphi}}=\frac{1}{2}\left(\partial_{t}+i \partial_{z}\right) .
$$


Using the general relations (2.21), as well as their corollaries

$$
1+2 L_{\varphi}=\frac{2 E_{\nu}}{1+E_{\nu}}, \quad 1-2 L_{\varphi}=\frac{2}{1+E_{\nu}}
$$

it is straightforward to rewrite the constraint (2.52) in terms of $\nu, \bar{\nu}$ and $E_{\nu}$, and to find that in the new setting this constraint is reduced to the pretty simple condition

$$
E_{\nu}=\frac{1}{2} \bar{\nu} \quad \Rightarrow \quad E^{S I}(\nu, \bar{\nu})=\frac{1}{2} \nu \bar{\nu}=\frac{1}{2} V^{2} \bar{V}^{2}=\frac{1}{2} a .
$$

Thus the BN model amounts to the simplest choice of the $O(2)$ invariant auxiliary interaction function $\mathcal{E}(a)$, the one quartic in the auxiliary tensor fields. This model was actually analyzed in detail in our paper [21] as the simplest example of the auxiliary interaction generating the non-polynomial self-dual electromagnetic Lagrangian in the $F$ representation. The relevant interaction in the $b$-representation is also linear,

$$
I^{S I}(b)=-2 b, \quad I_{b}^{S I}=-2 .
$$

Despite such a simple off-shell form of the auxiliary interaction, it is hardly possible to find a closed on-shell form of the nonlinear Lagrangian $L^{S I}(\varphi, \bar{\varphi})$, since the algebraic equations relating $a$ and $b$ to $\varphi, \bar{\varphi}$ are of the 5 -th order. E.g., the equation (2.38) becomes

$$
(b+1)^{2} \varphi \bar{\varphi}=b\left[\varphi+\bar{\varphi}+2(b-1)^{2}\right]^{2} .
$$

Nevertheless, it is straightforward to solve these equations as infinite series in $\varphi, \bar{\varphi}$ and then to restore $L^{B N}(\varphi, \bar{\varphi})$ to any order using the representations (2.31) or (2.39). In the present case it is simpler to solve the coupled set of the algebraic equations for the function $G(\varphi, \bar{\varphi})$ defined in (2.20):

$$
G=\frac{1}{1+\frac{1}{2} \bar{\nu}}=\frac{1}{1+\frac{1}{2} \bar{\varphi} \bar{G}^{2}}, \quad \bar{G}=\frac{1}{1+\frac{1}{2} \varphi G^{2}},
$$

which amounts to the following 5 -th order equation for $G$ :

$$
2\left(1+\frac{1}{2} \varphi G^{2}\right)^{2}=G\left[\bar{\varphi}+2\left(1+\frac{1}{2} \varphi G^{2}\right)^{2}\right]
$$

For the variables $a$ and $b$ one gets, respectively,

$$
a=\varphi \bar{\varphi} G^{2} \bar{G}^{2}, \quad b=\frac{1}{4} a .
$$

We give the self-dual Lagrangian $L^{S I}(\varphi, \bar{\varphi})$ up to 10 -th order in $F_{\alpha \beta}$ and $F_{\dot{\alpha} \dot{\beta}}$, in parallel with the analogous expansion of $L^{B I}(\varphi, \bar{\varphi})$. To this end, we use the expansion of $\mathcal{E}(a)$ in powers of $a$ up to the second order :

$$
\mathcal{E}(a)=e_{1} a+\frac{1}{2} e_{2} a^{2}+O\left(a^{3}\right), \quad \mathcal{E}_{a}=e_{1}+e_{2} a+O\left(a^{2}\right) .
$$


Here $e_{1}, e_{2}, \ldots$ are some real coefficients. All coefficients $e_{k}$ are non-vanishing for the BI model: $e_{1}=\frac{1}{2}, e_{2}=-\frac{1}{4}, \ldots$ 21], whereas all $e_{k}$ except for $e_{1}=\frac{1}{2}$ are vanishing for the SI model. Knowing $\mathcal{E}(a)$ up to the second order in $a$ allows one to uniquely restore $L^{s d}(\varphi, \bar{\varphi})$ up to the 10th order in $F_{\alpha \beta}, \bar{F}_{\dot{\alpha} \dot{\beta}}$ :

$$
\begin{aligned}
& L^{s d}=-\frac{1}{2}(\varphi+\bar{\varphi})+e_{1} \varphi \bar{\varphi}-e_{1}^{2}\left(\varphi^{2} \bar{\varphi}+\varphi \bar{\varphi}^{2}\right)+e_{1}^{3}\left(\varphi^{3} \bar{\varphi}+\varphi \bar{\varphi}^{3}\right)+\left(4 e_{1}^{3}+\frac{1}{2} e_{2}\right) \varphi^{2} \bar{\varphi}^{2} \\
& -e_{1}^{4}\left(\varphi^{4} \bar{\varphi}+\varphi \bar{\varphi}^{4}\right)-\left(10 e_{1}^{4}+2 e_{1} e_{2}\right)\left(\varphi^{3} \bar{\varphi}^{2}+\varphi^{2} \bar{\varphi}^{3}\right)+O\left(F^{12}\right) .
\end{aligned}
$$

The Lagrangian for the BI model corresponds to the choice $e_{1}=\frac{1}{2}, e_{2}=-\frac{1}{4}$ in (2.59) (c.f. (2.48)), while the Lagrangian of the SI (BN) model is reproduced with $e_{1}=\frac{1}{2}, e_{2}=0$. The Lagrangians for the $\mathrm{BI}$ and $\mathrm{BN}$ models as the power series in the tensor variables $t$ and $z$ were given in [18].

III. More examples. In [23] we also considered the following simple ansatz for the oneparameter deformation of the BI auxiliary Lagrangian (2.45)

$$
I_{b}=-\frac{2-c b}{(b-1)^{2}} .
$$

This ansatz gives rise to some 3 -rd order equation for $b$. The simplest choice is $c=2$, for which

$$
I_{b}=-2 /(1-b), \quad I(b)=2 \ln (1-b) .
$$

The corresponding $\varphi, \bar{\varphi}$ representation for the Lagrangian involves only one unknown function $r=\operatorname{Re} \mu$,

$$
\begin{aligned}
& \mu=r-\frac{(\varphi-\bar{\varphi})}{4}, \quad b=r^{2}-\frac{(\bar{\varphi}-\varphi)^{2}}{16}, \\
& L=-(\mu+\bar{\mu})+2 \ln (1-b)=-2 r-2 \ln \left[1-r^{2}+\frac{(\bar{\varphi}-\varphi)^{2}}{16}\right] .
\end{aligned}
$$

The basic algebraic equation in the $\mu$-representation (2.38) is reduced to the cubic equation for $r$ :

$$
r^{3}+(2+t) r^{2}+\left(1+\frac{1}{4} z^{2}\right) r-\frac{1}{2} t+\frac{1}{4}(2+t) z^{2}=0,
$$

where $t=(\varphi+\bar{\varphi}) / 2, z=(\varphi-\bar{\varphi}) / 2 i$ (see Eq. (A.10)). One can explicitly solve Eq. (2.63) using the Cardano formula or stick to its perturbative solution.

It is easy to obtain the $(F, V)$ representation (2.10) for the Lagrangian of the considered system. The corresponding $O(2)$ invariant function $\mathcal{E}(a)$ is given by the following expression

$$
\begin{aligned}
& \mathcal{E}(a)=2(\sqrt{1+a}-1)-2 \ln \left[\frac{1}{2}(1+\sqrt{1+a})\right]=\frac{1}{2} a-\frac{1}{16} a^{2}-\frac{1}{6} a^{3}+O\left(a^{4}\right), \\
& \mathcal{E}_{a}=\frac{1}{1+\sqrt{1+a}} .
\end{aligned}
$$

In 23] we also studied some duality-invariant systems which require solving the 4 -th order equations. 


\section{Auxiliary variables in duality-invariant theories with higher derivatives}

\subsection{Generalities}

The nonlinear electromagnetic Lagrangians with higher derivatives are functions of the variables

$$
F, \quad \partial_{m} F, \quad \partial_{m} \partial_{n} F, \quad \partial_{m} \partial_{n} \partial_{r} F \ldots
$$

and their complex conjugates. Derivatives of the field strengths appear with some coupling constants of non-trivial dimensions. The higher-derivative electromagnetic Lagrangians in the explicit form involve various scalar combinations of these variables, e.g.,

$$
F^{2}, \quad\left(\partial^{m} F \partial_{m} F\right),\left(\partial^{m} F^{2} \partial_{m} F^{2}\right), \quad\left(F \square^{N} F\right), \ldots .
$$

It is known that the higher-derivative generalizations of the duality-invariant Lagrangians contain all orders of derivatives of $F_{\alpha \beta}$ and $\bar{F}_{\dot{\alpha} \dot{\beta}}$ [17, 19].

The nonlinear equations of motion of such generalized Lagrangians are expressed through the Lagrange derivative [4]

$$
\begin{aligned}
& P_{\alpha \beta} \equiv\left(G^{+}\right)_{\alpha \beta}=\frac{1}{2}\left(\sum^{m n}\right)_{\alpha \beta} G_{m n}^{+}=i \frac{\Delta L}{\Delta F^{\alpha \beta}}, \\
& \frac{\Delta L}{\Delta F^{\alpha \beta}}=\frac{\partial L}{\partial F^{\alpha \beta}}-\partial_{m} \frac{\partial L}{\partial\left(\partial_{m} F^{\alpha \beta}\right)}+\partial_{m} \partial_{n} \frac{\partial L}{\partial\left(\partial_{m} \partial_{n} F^{\alpha \beta}\right)}+\ldots
\end{aligned}
$$

The definition of the $O(2)$ duality transformations and finding out the appropriate Lagrangians which would yield the duality-symmetric systems of equations with higher derivatives is a rather difficult task (see, e.g., [17, 19]). We propose to tackle this problem in the framework of the higher-derivative generalization of our formulation with the auxiliary bispinor fields.

This generalization is accomplished rather straightforwardly: we start from the original $(F, V)$ Lagrangian (2.10) with $O(2)$ invariant self-interaction $\mathcal{E}(\nu \bar{\nu})$ and allow the latter to depend also on the derivatives of auxiliary fields (still keeping invariance under the $O(2)$ transformations $(2.12))$ :

$$
\mathcal{L}\left(F, V, \partial V, \partial^{2} V, \ldots\right)=\mathcal{L}_{2}+\mathcal{E}\left(V, \partial V, \partial^{2} V, \ldots\right)
$$

Here $\mathcal{L}_{2}$ is the old bilinear part of the Lagrangian (2.11). By construction, this modified Lagrangian admits an analog of the GZ representation (2.27)

$$
\mathcal{L}(F, V, \ldots)=\frac{i}{2}[\bar{P}(F, V) \bar{F}-P(F, V) F]+V^{2}-(F V)+\bar{V}^{2}-(\bar{F} \bar{V})+\mathcal{E}(V, \ldots)
$$

for any $\mathcal{E}$. The equations of motion for this Lagrangian contain the Lagrange derivative of $\mathcal{E}$

$$
\begin{aligned}
& \partial_{\dot{\beta}}^{\alpha}(F-2 V)_{\alpha \beta}+\partial_{\beta}^{\dot{\alpha}}(\bar{F}-2 \bar{V})_{\dot{\alpha} \dot{\beta}}=0, \\
& F_{\alpha \beta}=V_{\alpha \beta}+\frac{1}{2} \frac{\Delta \mathcal{E}}{\Delta V^{\alpha \beta}} .
\end{aligned}
$$


It is easy to see that this set of equations together with the Bianchi identity (2.4) is covariant under the $O(2)$ duality transformations $(2.13),(2.12)$, provided that $\mathcal{E}(V, \partial V)$ is $O(2)$ invariant,

$$
\delta_{\omega} \mathcal{E}\left(V, \partial V, \partial^{2} V, \ldots\right)=0 .
$$

In the simplest non-trivial case, when the auxiliary interaction depends only on $V, \bar{V}$ and their first derivatives, i.e. $\mathcal{E}=\mathcal{E}(V, \partial V)$, we have

$$
\frac{\Delta \mathcal{E}}{\Delta V^{\alpha \beta}}=\frac{\partial \mathcal{E}}{\partial V^{\alpha \beta}}-\partial_{m} \frac{\partial \mathcal{E}}{\partial\left(\partial_{m} V^{\alpha \beta}\right)} .
$$

Using the definitions

$$
P_{\alpha \beta}(F, V)=i(F-2 V)_{\alpha \beta}, \quad \bar{P}_{\dot{\alpha} \dot{\beta}}(F, V)=-i(\bar{F}-2 \bar{V})_{\dot{\alpha} \dot{\beta}}
$$

and Eq.(3.8), we obtain the relations

$$
\begin{aligned}
& P^{2}+F^{2}=4(F V)-4 V^{2}=2 V^{\alpha \beta} \frac{\Delta \mathcal{E}}{\Delta V^{\alpha \beta}} \\
& =2 V^{\alpha \beta} \frac{\partial \mathcal{E}}{\partial V^{\alpha \beta}}+2 \partial_{m} V^{\alpha \beta} \frac{\partial \mathcal{E}}{\partial\left(\partial_{m} V^{\alpha \beta}\right)}-2 \partial_{m}\left[V^{\alpha \beta} \frac{\partial \mathcal{E}}{\partial\left(\partial_{m} V^{\alpha \beta}\right)}\right] .
\end{aligned}
$$

Now we can prove the integral form of the NGZ identity, using the $O(2)$ invariance of the interaction $\mathcal{E}(V, \partial V)$

$$
\int d^{4} x\left[P^{2}(F, V)+F^{2}-\bar{P}^{2}(F, V)-\bar{F}^{2}\right]=\int d^{4} x\left[\delta_{\omega} \mathcal{E}+\operatorname{div}\right]=0 .
$$

Substituting the solution of Eq.(3.8) into this relation, we obtain the nonlinear NGZ identity for the original $F$-representation with higher derivatives.

The same NGZ identity is valid in the general case, for an arbitrary $O(2)$ invariant auxiliary self-interaction $\mathcal{E}\left(V, \partial V, \partial^{2} V, \ldots\right)$.

Now we turn to the examples.

\subsection{The simplest case}

The simplest real invariant with two derivatives is

$$
\mathcal{E}(V, \partial V)=\rho=\bar{\rho} \sim \partial_{\beta}^{\dot{\beta}} V^{\alpha \beta} \partial_{\alpha}^{\dot{\xi}} \bar{V}_{\dot{\beta} \dot{\xi}}, \quad \delta_{\omega} \rho=0 .
$$

In the tensor representation the same $O(2)$ invariant reads as $\rho \sim \partial^{n} V_{m n}^{+} \partial_{r} V^{-r m}$. The relevant equations for $V_{\alpha \beta}$ and its conjugate are

$$
F_{\alpha \beta}=V_{\alpha \beta}+\frac{1}{2} b_{1} \partial_{\beta}^{\dot{\beta}} \partial_{\alpha}^{\dot{\rho}} \bar{V}_{\dot{\beta} \dot{\rho}}, \quad \bar{F}_{\dot{\alpha} \dot{\beta}}=\bar{V}_{\dot{\alpha} \dot{\beta}}+\frac{1}{2} b_{1} \partial_{\dot{\beta}}^{\beta} \partial_{\dot{\alpha}}^{\rho} V_{\beta \rho}
$$

where $b_{1}$ is a real constant. These equations have the non-local solution

$$
V_{\alpha \beta}=\frac{1}{1-\frac{1}{4} b_{1}^{2} \square^{2}}\left[F_{\alpha \beta}-\frac{1}{2} b_{1} \partial_{\beta}^{\dot{\beta}} \partial_{\alpha}^{\dot{\rho}} \bar{F}_{\dot{\beta} \dot{\rho}}\right] .
$$


The corresponding $L(\varphi, \bar{\varphi})$ is an extension of the standard Maxwell Lagrangian by nonlocal higher-derivative terms of the second order in the field strengths $F_{\alpha \beta}, \bar{F}_{\dot{\alpha} \dot{\beta}}$. Although such nonlocal modifications of the free bilinear action could appear as duality-invariant counterterms in extended supergravities [17], in what follows we assume that the higherderivative terms appear only at the interaction level, i.e. that the bilinear part of the full action still coincides with the standard Maxwell action. Under this natural assumption, $\mathcal{E}\left(V, \partial V, \partial^{2} V, \ldots\right)$ contains no terms bilinear in $V_{\alpha \beta}, \bar{V}_{\dot{\alpha} \dot{\beta}}$. The relevant auxiliary-field equations can always be solved by recursions, yielding no non-localities in the perturbative expansions and producing $L(\varphi, \bar{\varphi})$ in which all higher-derivative terms come out only in the interaction part.

In the remainder of this Section we will present a few specific examples of the dualityinvariant higher-derivative systems of this type.

\subsection{Auxiliary interaction with two derivatives}

As the first example we consider the simplest quartic mixed interaction with two derivatives

$$
\mathcal{E}_{(2)}=\frac{1}{2} \nu \bar{\nu}+c \partial^{m} \nu \partial_{m} \bar{\nu}, \quad \frac{\Delta \mathcal{E}_{(2)}}{\Delta V^{\alpha \beta}}=2 V_{\alpha \beta}\left[1+\frac{1}{2} \bar{\nu}-c \square \bar{\nu}\right],
$$

where $c$ is a coupling constant of dimension -2 and $\square=\partial^{m} \partial_{m}$. The auxiliary field equation has the form

$$
F_{\alpha \beta}=V_{\alpha \beta}\left(1+\frac{1}{2} \bar{\nu}-c \square \bar{\nu}\right), \quad \square \bar{\nu}=2\left(\bar{V}^{\dot{\alpha} \dot{\beta}} \square \bar{V}_{\dot{\alpha} \dot{\beta}}+\partial^{m} \bar{V}^{\dot{\alpha} \dot{\beta}} \partial_{m} \bar{V}_{\dot{\alpha} \dot{\beta}}\right) .
$$

The perturbative solution contains the higher derivatives

$$
\begin{aligned}
V_{\alpha \beta}^{(1)}= & F_{\alpha \beta}, \\
V_{\alpha \beta}^{(3)}= & -F_{\alpha \beta}\left(\frac{1}{2} \bar{\varphi}-c \square \bar{\varphi}\right), \\
V_{\alpha \beta}^{(5)}= & F_{\alpha \beta}\left\{\frac{1}{2} \bar{\varphi}\left(\frac{1}{2} \bar{\varphi}-c \square \bar{\varphi}\right)+\varphi\left(\frac{1}{2} \bar{\varphi}-c \square \bar{\varphi}\right)-c \square \bar{\varphi}\left(\frac{1}{2} \bar{\varphi}-c \square \bar{\varphi}\right)\right. \\
& \left.-2 c \square\left[\bar{\varphi}\left(\frac{1}{2} \varphi-c \square \varphi\right)\right]\right\}, \quad \text { etc. }
\end{aligned}
$$

The order of derivatives grows with each next recursion. The self-dual actions in the $F$-representation contains all orders of higher derivatives distributed over terms of all orders in the Maxwell field strengths. The perturbative construction of these actions, though being quite algorithmic, is much more involved technically as compared to the case without derivatives. For our model we obtain, up to terms of 8-th order,

$$
\begin{aligned}
L\left(F, \partial^{N} F\right)= & -\frac{1}{2}(\varphi+\bar{\varphi})+\frac{1}{2} \varphi \bar{\varphi}-\frac{1}{4} \varphi^{2} \bar{\varphi}-\frac{1}{4} \varphi \bar{\varphi}^{2}+c \partial^{m} \varphi \partial_{m} \bar{\varphi} \\
& +c \varphi \bar{\varphi}(\square \varphi+\square \bar{\varphi})-c^{2} \varphi(\square \bar{\varphi})^{2}-c^{2} \bar{\varphi}(\square \varphi)^{2}+O\left(F^{8}\right) .
\end{aligned}
$$

The model (3.17) can be regarded as the "minimal" higher-derivative deformation of the SI (or BN) model of Sect.3. Similarly, we could choose, as the undeformed $\mathcal{E}$, the 
interaction (2.49) corresponding to the BI theory. Adding to it the same term bilinear in derivatives as in (3.17), we would get the "minimal" higher-derivative duality-invariant deformation of the BI theory.

\subsection{Auxiliary interaction with four derivatives}

Simple examples of the duality-invariant systems with higher derivatives were analyzed in [17, 19], based on the "nonlinear twisted duality constraint" which is equivalent to our auxiliary field equation (3.8).

In our language these systems correspond to the quartic $O(2)$ invariant interactions

$$
A_{4}=A\left(\partial^{m} V \partial^{n} V\right)\left(\partial_{m} \bar{V} \partial_{n} \bar{V}\right), \quad B_{4}=B\left(\partial^{m} V \partial_{m} V\right)\left(\partial^{n} \bar{V} \partial_{n} \bar{V}\right),
$$

where $A$ and $B$ are some constants of dimension -4 and brackets denote traces with respect to the $S L(2, C)$ doublet indices.

We consider the construction of the Lagrangian in the $F$-representation for the model $A_{4}$. The basic equation (3.8) in this case is reduced to

$$
F_{\alpha \beta}=V_{\alpha \beta}-A \partial^{m}\left[\partial^{n} V_{\alpha \beta}\left(\partial_{m} \bar{V} \cdot \partial_{n} \bar{V}\right)\right] .
$$

The first two terms in its perturbative solution are as follows

$$
V_{\alpha \beta}^{(1)}=F_{\alpha \beta}, \quad V_{\alpha \beta}^{(3)}=A \partial^{m}\left[\partial^{n} F_{\alpha \beta}\left(\partial_{m} \bar{F} \cdot \partial_{n} \bar{F}\right)\right] .
$$

The corresponding Lagrangian in the $F$-representation involves higher derivatives, starting from the sixth order in fields

$$
L^{(6)}=\left(V^{(3)} V^{(3)}\right)-2 A\left[\left(V^{(3)} \partial^{n} \partial^{m} F\right)\left(\partial_{m} \bar{F} \partial_{n} \bar{F}\right)+\left(V^{(3)} \partial^{m} F\right) \partial^{n}\left(\partial_{m} \bar{F} \partial_{n} \bar{F}\right)\right]+\text { c.c. . }
$$

An alternative example of the auxiliary interaction with four derivatives which was not considered in [19] reads (just for a change, we use here the tensor notations, see Appendix A):

$$
C_{4}=C \partial^{n} V_{m n}^{+} \partial_{r} V^{-m r} \partial^{p} V_{q p}^{+} \partial_{s} V^{-q s} .
$$

Here $C$ is a constant of dimension -4 .

The basic auxiliary equation of this model reads

$$
F^{+m n}=V^{+m n}-2 C \pi_{u v}^{m n} \partial^{u}\left[\partial_{r} V^{-v r} \partial^{p} V_{q p}^{+} \partial_{s} V^{-q s}\right]
$$

where

$$
\pi_{u v}^{m n}=\frac{1}{4}\left(\delta_{u}^{m} \delta_{v}^{n}-\delta_{v}^{m} \delta_{u}^{n}+i \varepsilon_{u v}^{m n}\right)
$$

is the projector on the self-dual part. The equation for $F_{m n}^{-}$can be obtained by complex conjugation. The first two terms of the perturbative solution are given by the expressions

$$
V_{m n}^{+(1)}=F_{m n}^{+}, \quad V_{m n}^{+(3)}=2 C \pi_{m n}^{u v} \partial_{u}\left[\partial^{r} F_{v r}^{-} \partial^{p} F_{q p}^{+} \partial_{s} F^{-q s}\right] .
$$

The perturbative construction of the Lagrangian in the $F$-representation is completely analogous to the previous cases. 


\section{Conclusions}

In this paper, following and extending our earlier results [21, 23], we presented the evidence that all duality-invariant systems of nonlinear electrodynamics (including those with higher derivatives) admit an off-shell formulation with the auxiliary bispinor (tensorial) fields. These fields are fully unconstrained off shell, there is no need to express them through any second gauge potentials, etc.

The whole information about the given duality-invariant system is encoded in the $O(2)$ invariant interaction function which depends only on the auxiliary fields (or also on their derivatives) and can be chosen at will. In many cases it looks much simpler compared to the final on-shell action written in terms of the Maxwell field strengths. The renowned nonlinear NGZ constraint is linearized in the new formulation and becomes just the requirement of $O(2)$ invariance of the auxiliary interaction. The $O(2)$ (and in fact $U(N)[22,25])$ duality symmetry is realized off shell by linear transformations.

The basic algebraic equations eliminating the auxiliary tensor fields are equivalent to the recently employed "nonlinear twisted self-duality constraint" [17, 18, 19]. In our approach this constraint appears as the equations of motion (2.26), (3.8) associated with the well defined off-shell Lagrangians. Based on the relations (A.24) - (A.27), it is instructive here to give the dictionary of correspondence between the notations used in [18, 19] and our bispinor notation (up to numerical coefficients)

$$
\left(T_{m n}^{+}, T_{m n}^{*-}\right) \Leftrightarrow\left[(F-V)_{\alpha \beta},(\bar{F}-\bar{V})_{\dot{\alpha} \dot{\beta}}\right], \quad\left(T_{m n}^{*+}, T_{m n}^{-}\right) \Leftrightarrow\left(V_{\alpha \beta}, \bar{V}_{\dot{\alpha} \dot{\beta}}\right), \quad \mathcal{I}^{(1)} \Leftrightarrow \mathcal{E} .
$$

Let us finish by mentioning a few proposals for the future study.

First, it is of urgent interest to extend our formulation to the $\mathcal{N}=1, \mathcal{N}=2, \ldots$ supersymmetric duality-invariant systems, including supersymmetric Born-Infeld theories, in both the flat and the supergravity backgrounds.

Second, it is desirable to learn how the scalar and other fields can be self-consistently inscribed into the framework with the auxiliary tensorial fields.

Finally, we would like to point out that the formulation with the auxiliary fields suggests a new view of the duality-invariant systems: it seems natural, in both the classical and the quantum cases, not to eliminate the tensorial auxiliary fields by their equations of motion beforehand, but to deal with the off-shell actions at all steps. This would as well refer to the quantum counterterms which should appear in this approach as corrections to the original auxiliary interaction function $\mathcal{E}$. It is worthwhile here to recall the off-shell superfield approach to supersymmetric theories, which in many cases radically facilitates the quantum calculations and unveils the relevant intrinsic geometric properties without any need to pass on shell through eliminating the auxiliary fields. It is also worth recalling that the tensorial auxiliary fields have originally appeared just within the off-shell superfield formulation of $\mathcal{N}=3$ supersymmetric Born-Infeld theory [21]. 


\section{Acknowledgements}

We acknowledge a partial support from the RFBR grants Nr.12-02-00517, Nr.11-02-90445, the grant DFG LE 838/12-1 and a grant from the Heisenberg-Landau program.

\section{A. Spinor and tensor notations in electrodynamics}

We use the Minkowski metric $\eta_{m n}=\operatorname{diag}(1,-1,-1,-1)$ and the $2 \times 2$ Weyl matrices

$$
\begin{aligned}
& \left(\sigma^{m}\right)_{\alpha \dot{\beta}}, \quad\left(\bar{\sigma}^{m}\right)^{\dot{\alpha} \beta}=\varepsilon^{\beta \alpha} \varepsilon^{\dot{\alpha} \dot{\beta}}\left(\sigma^{m}\right)_{\alpha \dot{\beta}}, \\
& \left(\sigma^{m} \bar{\sigma}^{n}+\sigma^{n} \bar{\sigma}^{m}\right)_{\alpha}^{\beta}=2 \eta^{m n} \delta_{\alpha}^{\beta}, \\
& \sigma^{n} \bar{\sigma}^{s} \sigma^{m}=\eta^{n s} \sigma^{m}-\eta^{n m} \sigma^{s}+\eta^{s m} \sigma^{n}-i \varepsilon^{n s m r} \sigma_{r} .
\end{aligned}
$$

The vectors in the spinor and tensor notations are related as

$$
A_{\alpha \dot{\beta}}=\left(\sigma^{m}\right)_{\alpha \dot{\beta}} A_{m}, \quad \partial_{\alpha \dot{\beta}}=\left(\sigma^{m}\right)_{\alpha \dot{\beta}} \partial_{m} .
$$

The same correspondence for the Maxwell field strengths is presented by the relations

$$
\begin{aligned}
F_{\alpha}^{\beta}(A) \equiv & \frac{1}{4} \partial_{\alpha \dot{\beta}} A^{\dot{\beta} \beta}-\frac{1}{4} \partial^{\dot{\beta} \beta} A_{\alpha \dot{\beta}}=\frac{1}{8}\left(\sigma^{m} \bar{\sigma}^{n}-\sigma^{n} \bar{\sigma}^{m}\right)_{\alpha}^{\beta} F_{m n} \\
& =\frac{1}{8}\left(\sigma^{m} \bar{\sigma}^{n}-\sigma^{n} \bar{\sigma}^{m}\right)_{\alpha}^{\beta} F_{m n}^{+}, \\
\bar{F}_{\dot{\alpha}}^{\dot{\beta}}= & \frac{1}{4}\left(\bar{\sigma}^{n}\right)^{\dot{\beta} \beta}\left(\sigma^{m}\right)_{\beta \dot{\alpha}} F_{m n}=-\frac{1}{8}\left(\bar{\sigma}^{m} \sigma^{n}-\bar{\sigma}^{n} \sigma^{m}\right)_{\dot{\alpha}}^{\dot{\beta}} F_{m n} \\
& =-\frac{1}{8}\left(\bar{\sigma}^{m} \sigma^{n}-\bar{\sigma}^{n} \sigma^{m}\right)_{\dot{\alpha}}^{\dot{\beta}} F_{m n}^{-},
\end{aligned}
$$

where

$$
\begin{aligned}
& F_{m n}=\partial_{m} A_{n}-\partial_{n} A_{m}, \quad \tilde{F}_{m n}=\frac{1}{2} \varepsilon_{m n r s} F^{r s}, \quad F_{m n}^{+}=\frac{1}{2} F_{m n}+\frac{i}{2} \tilde{F}_{m n}, \\
& \widetilde{F+}_{m n}=-i F_{m n}^{+}, \quad F_{m n}^{-}=\frac{1}{2} F_{m n}-\frac{i}{2} \tilde{F}_{m n}, \quad \widetilde{F^{-}}{ }_{m n}=i F_{m n}^{-} .
\end{aligned}
$$

Thus, $F_{\alpha \beta}$ is the equivalent bispinor notation for the self-dual tensor field $F_{m n}^{+}$, and $\bar{F}_{\dot{\alpha} \dot{\beta}}$ amounts to the anti-self-dual tensor $F_{m n}^{-}$.

The scalar variables in the spinor formalism are related to the analogous variables in the tensor formalism as

$$
\begin{aligned}
& \varphi=F^{\alpha \beta} F_{\alpha \beta}=t+i z=\frac{1}{2} F^{+m n} F_{m n}^{+}, \\
& \bar{\varphi}=\bar{F}^{\dot{\alpha} \dot{\beta}} \bar{F}_{\dot{\alpha} \dot{\beta}}=t-i z=\frac{1}{2} F^{-m n} F_{m n}^{-}, \\
& t=\frac{1}{4} F^{m n} F_{m n}=\frac{1}{2}(\varphi+\bar{\varphi}), \quad z=\frac{1}{4} F^{m n} \tilde{F}_{m n}=\frac{1}{2 i}(\varphi-\bar{\varphi}), \\
& \frac{\partial}{\partial \varphi}=\frac{1}{2} \partial_{t}-\frac{i}{2} \partial_{z}, \quad \frac{\partial}{\partial \bar{\varphi}}=\frac{1}{2} \partial_{t}+\frac{i}{2} \partial_{z} .
\end{aligned}
$$


The bispinor and tensor representations of the dual field strengths appearing in the nonlinear equations of motion are related as

$$
\begin{aligned}
& P_{\alpha}^{\beta}(F)=\frac{1}{8}\left(\sigma^{m} \bar{\sigma}^{n}-\sigma^{n} \bar{\sigma}^{m}\right)_{\alpha}^{\beta} G_{m n}^{+}, \quad \bar{P}_{\dot{\alpha}}^{\dot{\beta}}=-\frac{1}{8}\left(\bar{\sigma}^{m} \sigma^{n}-\bar{\sigma}^{n} \sigma^{m}\right)_{\dot{\alpha}}^{\dot{\beta}} G_{m n}^{-}, \\
& G_{m n}^{ \pm}=\frac{1}{2} G_{m n} \pm \frac{i}{2} \tilde{G}_{m n}, \quad \tilde{G}_{m n}=2 \frac{\Delta L}{\Delta F^{m n}}, \quad G_{m n}=-\varepsilon_{m n r s} \frac{\Delta L}{\Delta F_{r s}}
\end{aligned}
$$

where we employed the Lagrange derivatives.

The similar relations are valid for the auxiliary fields

$$
V_{\alpha}^{\beta}=\frac{1}{8}\left(\sigma^{m} \bar{\sigma}^{n}-\sigma^{n} \bar{\sigma}^{m}\right)_{\alpha}^{\beta} V_{m n}^{+}, \quad \bar{V}_{\dot{\alpha}}^{\dot{\beta}}=-\frac{1}{8}\left(\bar{\sigma}^{m} \sigma^{n}-\bar{\sigma}^{n} \sigma^{m}\right)_{\dot{\alpha}}^{\dot{\beta}} V_{m n}^{-} .
$$

The complex auxiliary tensorial fields $V_{m n}^{ \pm}$are expressed through the real fields as

$$
\begin{aligned}
& V_{m n}^{+}=\frac{1}{2} V_{m n}+\frac{i}{2} \tilde{V}_{m n}, \quad V_{m n}^{-}=\frac{1}{2} V_{m n}-\frac{i}{2} \tilde{V}_{m n}, \\
& \tilde{\tilde{V}}_{m n}=-V_{m n}, \quad \tilde{V}^{m n} \tilde{V}_{m n}=-V^{m n} V_{m n} .
\end{aligned}
$$

The scalar variable $\nu$ can be represented as

$$
\begin{aligned}
& \nu=V^{\alpha \beta} V_{\alpha \beta}=v+i w, \quad v=\frac{1}{4} V^{m n} V_{m n}, \quad w=\frac{1}{4} \tilde{V}^{m n} V_{m n}, \\
& \partial_{\nu}=\frac{1}{2} \partial_{v}-\frac{i}{2} \partial_{w} .
\end{aligned}
$$

The real tensor and scalar fields just introduced have the following $O(2)$ transformation laws

$$
\begin{aligned}
& \delta_{\omega} V_{m n}=\omega \tilde{V}_{m n}, \quad \delta_{\omega} \tilde{V}_{m n}=-\omega V_{m n}, \\
& \delta_{\omega} v=2 \omega w, \quad \delta_{\omega} w=-2 \omega v .
\end{aligned}
$$

In the tensorial notations our basic $O(2)$ invariant variable $a$ is reduced to

$$
a=\nu \bar{\nu}=v^{2}+w^{2} .
$$

The one-to-one correspondence between the specific variables used in [17, 18, 19] and our variables in the tensor notation is as follows 3 :

$$
\begin{aligned}
& T=F-i G=F-2 i \tilde{V}+i \tilde{F}, \quad \tilde{T}=\tilde{F}-i \tilde{G}=\tilde{F}+2 i V-i F \\
& T^{*}=F+i G=F+2 i \tilde{V}-i \tilde{F}, \quad \widetilde{T^{*}}=\tilde{F}-2 i V+i F \\
& \delta_{\omega} T=i \omega T, \quad \delta_{\omega} \tilde{T}=i \omega \tilde{T}, \quad \delta_{\omega} T^{*}=-i \omega T^{*}, \quad \delta_{\omega} \widetilde{T^{*}}=-i \omega \widetilde{T^{*}}
\end{aligned}
$$

\footnotetext{
${ }^{3}$ Sometimes, for brevity, we omit the antisymmetric tensor indices.
} 
Here Eqs.(A.29) and (A.30) were used. The relations between self-dual (and anti-self-dual) parts of these two sets of complex variables can be collected as

$$
\begin{aligned}
& T^{+}=\frac{1}{2}(T+i \tilde{T})=(F-V)+i(\tilde{F}-\tilde{V})=2 F^{+}-2 V^{+}, \\
& T^{-}=\frac{1}{2}(T-i \tilde{T})=V-i \tilde{V}=2 V^{-} \\
& T^{*+} \equiv \bar{T}^{+}=\left(T^{-}\right)^{*}=\frac{1}{2}\left(T^{*}+i \widetilde{T^{*}}\right)=V+i \tilde{V}=2 V^{+} \\
& T^{*-} \equiv \bar{T}^{-}=\left(T^{+}\right)^{*}=\frac{1}{2}\left(T^{*}-i \widetilde{T^{*}}\right)=F-i \tilde{F}-V+i \tilde{V}=2 F^{-}-2 V^{-} .
\end{aligned}
$$

Being cast in the tensor notations, our Lagrangian (2.10) becomes:

$$
\begin{aligned}
& \mathcal{L}(F, V)=-\frac{1}{4} F^{m n} F_{m n}+\frac{1}{2}\left(V^{m n}-F^{m n}\right)\left(V_{m n}-F_{m n}\right)+\mathcal{E}\left(v^{2}+w^{2}\right) \\
& =-\frac{1}{4}\left[\left(F^{+}\right)^{2}+\left(F^{-}\right)^{2}\right]+\frac{1}{2}\left[\left(V^{+}-F^{+}\right)^{2}+\left(V^{-}-F^{-}\right)^{2}\right]+\mathcal{E}\left(v^{2}+w^{2}\right)
\end{aligned}
$$

and $v^{2}+w^{2}=\frac{1}{4}\left(V^{+}\right)^{2}\left(V^{-}\right)^{2}$. The dual nonlinear field strength $\tilde{G}^{m n}$ is expressed through our variables as

$$
\tilde{G}^{m n}(F, V)=2 \frac{\partial \mathcal{L}(V, F)}{\partial F_{m n}}=(F-2 V)^{m n} .
$$

Applying the tilde operation to both sides of this equality yields

$$
G^{m n}(F, V)=(2 \tilde{V}-\tilde{F})^{m n} .
$$

In terms of self-dual components, the same relations read

$$
\begin{aligned}
G^{+} & =\frac{1}{2}(G+i \tilde{G})=i F^{+}-2 i V^{+}, & V^{+}=\frac{1}{2}\left(F^{+}+i G^{+}\right), \\
G^{-} & =\frac{1}{2}(G-i \tilde{G})=-i F^{-}+2 i V^{-}, & V^{-}=\frac{1}{2}\left(F^{-}-i G^{-}\right) .
\end{aligned}
$$

The auxiliary equations of our formalism (2.17) are completely equivalent to the "twisted nonlinear self-duality condition" of Refs. [17, 18].

The tensor version of our algebraic equation reads

$$
\left(F^{+}-V^{+}\right)_{m n}=\frac{\partial \mathcal{E}(a)}{\partial V^{+m n}}=\frac{1}{2} V_{m n}^{+}\left(V^{-}\right)^{2} \mathcal{E}_{a} .
$$

After passing to the $T$-tensor notation by Eqs. (A.25), (A.26), the same equation is rewritten as

$$
T_{m n}^{+}=4 \frac{\partial \mathcal{E}(a)}{\partial \bar{T}^{+m n}}=\frac{1}{8}\left(\bar{T}^{+}\right)_{m n}\left(T^{-}\right)^{2} \mathcal{E}_{a}, \quad a=\frac{1}{4}\left(V^{+}\right)^{2}\left(V^{-}\right)^{2}=\frac{1}{64}\left(\bar{T}^{+}\right)^{2}\left(T^{-}\right)^{2},
$$

that precisely coincides with the general twisted self-duality condition. Our interaction function $\mathcal{E}$ proves to be identical to the "deformation function" $\mathcal{I}^{(1)}$ used in [18, 19]. The general case with derivatives on the Maxwell field strengths corresponds to passing to the Lagrange derivatives in (A.32) and (A.33). 


\section{B. Schrödinger constraint and BI theory}

Similarly to [2, 18, we consider here the Schrödinger type 24] nonlinear constraints

$$
T_{m n} T^{r s} \tilde{T}_{r s}-\tilde{T}_{m n} T^{r s} T_{r s}= \pm \frac{1}{8} \tilde{T}_{m n}^{*}\left(T^{r s} \tilde{T}_{r s}\right)^{2} .
$$

The contraction of this condition with $T^{m n}$ yields the NGZ self-duality constraint

$$
T^{m n} \tilde{T}_{m n}^{*}=F^{m n} \tilde{F}_{m n}+G^{m n} \tilde{G}_{m n}=0
$$

for both signs in the right-hand side of (B.1).

For definiteness we choose the sign minus and, using the relations from the Appendix A, equivalently rewrite (B.1) in the bispinor notations as the two conditions

$$
\begin{aligned}
& 2(F-V)_{\alpha \beta} \bar{V}^{2}=V_{\alpha \beta}\left[\bar{V}^{2}-(F-V)^{2}\right]^{2}, \\
& 2 \bar{V}_{\dot{\alpha} \dot{\beta}}(F-V)^{2}=(\bar{F}-\bar{V})_{\dot{\alpha} \dot{\beta}}\left[\bar{V}^{2}-(F-V)^{2}\right]^{2} .
\end{aligned}
$$

Now we substitute the twisted self-duality equation (A.32) in its spinorial form (2.26) into these constraints and find that the latter imply the following equation for the auxiliary interaction

$$
2 \mathcal{E}_{a}=\left[1-a \mathcal{E}_{a}^{2}\right]^{2} .
$$

This equation is greatly simplified in terms of the variable $b$ and the interaction $I(b)$ defined in (2.36):

$$
I_{b}=-\frac{2}{(b-1)^{2}} \rightarrow I(b)=\frac{2 b}{b-1} .
$$

It is recognized as the interaction defining the BI theory.

The sign plus in the constraint (B.1) corresponds to the "twisted" solution $E(a) \rightarrow$ $-E(a)$ which amounts to the choice of the opposite sign of the BI coupling constant, $f^{2} \rightarrow-f^{2}$. These two options exhaust all possible solutions of the constraints (B.1).

\section{Manifestly duality-invariant action with auxiliary fields}

It is known that the duality-invariant systems admit an alternative description in which the $O(2)$ duality symmetry becomes manifest at cost of loosing the manifest Lorentz symmetry (see, e.g., [11, 20] ) 4 . Here we demonstrate that the formulation with the auxiliary tensorial fields can also be rearranged in a similar way.

\footnotetext{
${ }^{4}$ Both symmetries can be made explicit after introducing some additional gauge degrees of freedom [10; here we do not touch this type of theories.
} 
We start by introducing the $3 D$ notations for the Maxwell field strength $F_{m n}(A)=$ $\partial_{m} A_{n}-\partial_{n} A_{m}$ and the auxiliary fields $V_{m n}$ :

$$
\begin{aligned}
& E_{k}(A):=F_{0 k}(A)=\partial_{0} A_{k}-\partial_{k} A_{0}, \quad \varepsilon_{k l j} B_{j}:=F_{k l}(A)=\partial_{k} A_{l}-\partial_{l} A_{k}, \\
& \tilde{E}_{k}=B_{k}, \quad \tilde{B}_{k}=-E_{k}, \quad \tilde{F}_{k l}=-\varepsilon_{k l j} E_{j}, \\
& V_{k}:=V_{0 k}, \quad \varepsilon_{k l j} U_{j}:=V_{k l},
\end{aligned}
$$

where $k, l=1,2,3$. In the new setting, the scalar combinations of the auxiliary fields are rewritten as

$$
\begin{aligned}
& \nu=v+i w=\frac{1}{2} U_{k} U_{k}-\frac{1}{2} V_{k} V_{k}-i V_{k} U_{k}, \\
& a(V, U)=v^{2}+w^{2}=\frac{1}{4}\left(U_{i} U_{i}\right)^{2}+\frac{1}{4}\left(V_{i} V_{i}\right)^{2}-\frac{1}{2}\left(U_{i} U_{i}\right)\left(V_{k} V_{k}\right)+\left(V_{i} U_{i}\right)^{2} .
\end{aligned}
$$

Next, we introduce one more gauge potential $A_{m}^{\prime}$, as well as an extra independent auxiliary field $N_{m n}=-N_{n m}$, and rewrite our Lagrangian (2.10) with $E_{(\nu, \bar{\nu})}=\mathcal{E}(a)$ in the equivalent form:

$$
\mathcal{L}(F, V, A, B)=\frac{1}{4} \hat{F}^{m n} \hat{F}_{m n}-\hat{F}^{m n} V_{m n}+\frac{1}{2} V^{m n} V_{m n}-\frac{1}{4} \varepsilon^{m n r s} N_{m n} F_{r s}^{\prime}+\mathcal{E}\left(v^{2}+w^{2}\right),(\mathrm{C}
$$

where $\hat{F}_{m n}=N_{m n}+F_{m n}(A)$ and $F_{r s}^{\prime}=\partial_{r} A_{s}^{\prime}-\partial_{s} A_{r}^{\prime}$. The field $A_{m}^{\prime}$ serves as the Lagrange multiplier producing the Bianchi identity for $N_{m n}$, which can be solved as $N_{m n}=\partial_{m} C_{n}-$ $\partial_{n} C_{m}$. Then $\hat{F}_{m n}=\partial_{m}\left(C_{n}+A_{n}\right)-\partial_{n}\left(C_{m}+A_{m}\right)$, which takes us back to the original Lagrangian (2.10).

On the other hand, following [11, we can keep both gauge fields in the Lagrangian (C.5), in which case it displays the new vectorial gauge symmetry

$$
\delta N_{m n}=\partial_{m} \lambda_{n}-\partial_{n} \lambda_{m}, \quad \delta A_{m}=-\lambda_{m} .
$$

We can make use of this gauge freedom to fix the magnetic non-covariant gauge

$$
N_{0 k}=0 .
$$

It leaves us with $N_{k l}=\varepsilon_{k l j} N_{j}$ and implies

$$
\frac{1}{2} N^{m n} N_{m n}=N_{k} N_{k}, N^{m n} V_{m n}=2 N_{k} U_{k}, \frac{1}{2} \tilde{N}^{m n} F_{m n}^{\prime}=-N_{k} E_{k}^{\prime}, \frac{1}{2} N^{m n} F_{m n}=N_{k} B_{k} .
$$

In the gauge (C.7), the bilinear part of our Lagrangian reads:

$$
\begin{aligned}
\mathcal{L}_{2}\left(N, V, U, A, A^{\prime}\right)= & \frac{1}{2} N_{k} N_{k}-N_{k}\left(2 U_{k}-B_{k}-E_{k}^{\prime}\right)-\frac{1}{2} E_{k} E_{k}+\frac{1}{2} B_{k} B_{k} \\
& +2 E_{k} V_{k}-2 B_{k} U_{k}-V_{k} V_{k}+U_{k} U_{k} .
\end{aligned}
$$

After eliminating the auxiliary field $N_{k}$ by the equation of motion,

$$
N_{k}=2 U_{k}-B_{k}-E_{k}^{\prime},
$$


the whole $O(2)$ invariant Lagrangian with two gauge potentials and the auxiliary fields $V_{k}, U_{k}$ is finally written as

$$
\begin{aligned}
\mathcal{L}\left(A, A^{\prime}, V, U\right)= & -\frac{1}{2} E_{k} E_{k}-\frac{1}{2} E_{k}^{\prime} E_{k}^{\prime}+B_{k} E_{k}^{\prime}+2 E_{k} V_{k}+2 U_{k} E_{k}^{\prime} \\
& -V_{k} V_{k}-U_{k} U_{k}+\mathcal{E}[a(V, U)]
\end{aligned}
$$

The $O(2)$ duality transformations are realized on these fields as

$$
\begin{aligned}
& \delta_{\omega} V_{k}=\omega U_{k}, \quad \delta_{\omega} U_{k}=-\omega V_{k}, \\
& \delta_{\omega} E_{k}=\omega E_{k}^{\prime}, \quad \delta_{\omega} E_{k}^{\prime}=-\omega E_{k}, \quad \delta_{\omega} B_{k}=\omega B_{k}^{\prime}, \quad \delta_{\omega} B_{k}^{\prime}=-\omega B_{k} .
\end{aligned}
$$

Then, using the relations

$$
E_{k} B_{k}=-\frac{1}{4} F^{m n}(A) \tilde{F}_{m n}(A)=\operatorname{div}, \quad E_{k}^{\prime} B_{k}^{\prime}=-\frac{1}{4} F^{m n}\left(A^{\prime}\right) \tilde{F}_{m n}\left(A^{\prime}\right)=\operatorname{div},
$$

we prove the $O(2)$ invariance of the non-covariant action

$$
\delta S=\int d^{4} x \delta L\left(A, A^{\prime}, V, U\right)=\omega \int d^{4} x\left(E_{k}^{\prime} B_{k}^{\prime}-E_{k} B_{k}\right)=0 .
$$

The twisted self-duality equations have the following form in this formalism:

$$
\begin{aligned}
V_{k} & =E_{k}+\frac{1}{2} \mathcal{E}_{a}\left\{V_{k}\left[\left(V_{l} V_{l}\right)-\left(U_{l} U_{l}\right)\right]+2 U_{k}\left(V_{l} U_{l}\right)\right\} \\
U_{k} & =E_{k}^{\prime}+\frac{1}{2} \mathcal{E}_{a}\left\{U_{k}\left[\left(U_{l} U_{l}\right)-\left(V_{l} V_{l}\right)\right]+2 V_{k}\left(V_{l} U_{l}\right)\right\} \\
\mathcal{E}_{a} & =\frac{1}{2}+e_{2} a+\ldots
\end{aligned}
$$

These algebraic equations can be solved perturbatively in terms of the fields strengths $E_{k}$ and $E_{k}^{\prime}$. To the lowest order:

$$
\begin{aligned}
& V_{k}=E_{k}+\frac{1}{4}\left\{E_{k}\left[\left(E_{l} E_{l}\right)-\left(E_{l}^{\prime} E_{l}^{\prime}\right)\right]+2 E_{k}^{\prime}\left(E_{l} E_{l}^{\prime}\right)\right\}+O\left(E^{5}\right), \\
& U_{k}=E_{k}^{\prime}+\frac{1}{4}\left\{E_{k}^{\prime}\left[\left(E_{l}^{\prime} E_{l}^{\prime}\right)-\left(E_{l} E_{l}\right)\right]+2 E_{k}\left(E_{l} E_{l}^{\prime}\right)\right\}+O\left(E^{5}\right) .
\end{aligned}
$$

After substituting these expressions back into (C.10), we obtain the manifestly self-dual Lagrangian as a function of the $3 D$ field strengths

$$
\begin{aligned}
L^{s d}\left(A, A^{\prime}\right)= & \frac{1}{2} E_{k} E_{k}+\frac{1}{2} E_{k}^{\prime} E_{k}^{\prime}+B_{k} E_{k}^{\prime}+\frac{1}{8}\left(E_{k} E_{k}\right)^{2}+\frac{1}{8}\left(E_{k}^{\prime} E_{k}^{\prime}\right)^{2} \\
& -\frac{1}{8}\left(E_{k} E_{k}\right)\left(E_{l}^{\prime} E_{l}^{\prime}\right)+\frac{1}{2}\left(E_{k} E_{k}^{\prime}\right)^{2}+\ldots
\end{aligned}
$$

The magnetic version of the manifestly $O(2)$ invariant action can be obtained through the discrete duality transformation of both fields $E \rightarrow B, \quad B \rightarrow-E$. 


\section{References}

[1] M.K. Gaillard and B. Zumino, Duality rotations for interacting fields, Nucl. Phys. B 193 (1981) 221.

[2] M.K. Gaillard and B. Zumino, Self-duality in nonlinear electromagnetism, In: Supersymmetry and quantum field theory, eds. J. Wess and V.P. Akulov, p. 121-129, Springer-Vellag, 1998, hep-th/9705226;

M.K. Gaillard and B. Zumino, Nonlinear electromagnetic self-duality and Legendre transform, In: Duality and Supersymmetric Theories, eds. D.I. Olive and P.C. West, p. 33, Cambridge University Press, 1999, hep-th/9712103.

[3] G.W. Gibbons and D.A. Rasheed, Electric-magnetic duality rotations in nonlinear electrodynamics, Nucl. Phys. B 454 (1995) 185, hep-th/9506035.

[4] S.M. Kuzenko and S. Theisen, Nonlinear self-duality and supersymmetry, Fortsch. Phys. 49 (2001) 273, hep-th/0007231.

[5] S. Deser, C. Teitelboim, Duality transformations of abelian and nonabelian gauge fields, Phys. Rev. D 13 (1976) 1592.

[6] M. Henneaux, C. Teitelboim, Dynamics of chiral (selfdual) p-forms, Phys. Lett. B 206 (1988) 650.

[7] J.H. Schwarz, A. Sen, Duality symmetric actions, Nucl. Phys. B 411 (1994) 35, hep-th/9304154.

[8] M. Hatsuda, K. Kamimura and S. Sekia, Electric-magnetic duality invariant Lagrangians, Nucl. Phys. B 561 (1999) 341, hep-th/9906103.

[9] X. Bekaert and S. Cucu, Deformations of duality-symmetric theories, Nucl. Phys. B 610 (2001) 433, hep-th/0104048.

[10] P. Pasti, D. Sorokin and M. Tonin, Duality symmetric actions with manifest spacetime symmetries, Phys. Rev. D 52 (1995) R4277, hep-th/9506109;

P. Pasti, D. Sorokin and M. Tonin, Covariant actions for models with non-linear twisted self-duality, Phys.Rev. D 86 (2012) 045013, arXiv:1205.4243 [hep-th].

[11] M. Roček and A. Tseytlin, Partial breaking of global D=4 supersymmetry, constrained superfields, and 3-brane actions, Phys. Rev. D 59 (1999) 106001, hep-th/9811232.

[12] A.A. Tseytlin, Born-Infeld action, supersymmetry and string theory, In: The many faces of superworld, Yu. Golfand memorial volume, ed. M.A. Shifman, p. 417, World Scientific, 2000, hep-th/9908105.

[13] P. Aschieri, D. Brace, B. Morariu, B. Zumino, Proof of a symmetrized trace conjecture for the abelian Born-Infeld Lagrangian, Nucl. Phys. B 588 (2000) 521, hep-th/0003228. 
[14] P. Aschieri, S. Ferrara and B. Zumino, Duality rotations in nonlinear electrodynamics and extended supergravity, Riv. Nuovo Cim. 31 (2008) 625, arXiv:0807.4039 [hep-th].

[15] G. Bossard, C. Hillmann, H. Nicolai, $E_{7(7)}$ symmetry in perturbatively quantised $\mathcal{N}=$ 8 supergravity, JHEP 1012 (2010) 052, arXiv:1007.5472 [hep-th].

[16] R. Kallosh, $E_{7(7)}$ symmetry and finiteness of $\mathcal{N}=8$ supergravity, arXiv:1103.4115 [hep-th]; R. Kallosh, $\mathcal{N}=8$ counterterms and $E_{7(7)}$ current conservation, JHEP 1106 (2011) 073, arXiv:1104.5480 [hep-th].

[17] G. Bossard, H. Nicolai, Counterterms vs. dualities, JHEP 1108 (2011) 074, arXiv:1105.1273 [hep-th].

[18] J.J.M. Carrasco, R. Kallosh, R. Roiban, Covariant procedure for perturbative nonlinear deformation of duality-invariant theories, Phys. Rev. D 85 (2012) 025007, arXiv:1108.4390 [hep-th].

[19] W. Chemissany, R. Kallosh, T. Ortin, Born-Infeld with higher derivatives, Phys. Rev. D 85 (2012) 046002, arXiv:1112.0332 [hep-th].

[20] R. Roiban, A. Tseytlin, On duality symmetry in perturbative quantum theory, JHEP 1210 (2012) 099, arXiv:1205.0176 [hep-th].

[21] E.A. Ivanov, B.M. Zupnik, $\mathcal{N}=3$ supersymmetric Born-Infeld theory, Nucl. Phys. B 618 (2001) 3, hep-th/0110074.

[22] E.A. Ivanov, B.M. Zupnik, New representation for Lagrangians of self-dual nonlinear electrodynamics, In: Supersymmetries and quantum symmetries, eds. E. Ivanov et al, p. 235, Dubna, 2002, hep-th/0202203.

[23] E.A. Ivanov, B.M. Zupnik, New approach to nonlinear electrodynamics: dualities as symmetries of interaction, Yadern. Fiz. 67 (2004) 2212; [Phys. Atom. Nucl. 67 (2004) 2188], hep-th/0303192.

[24] E. Schrödinger, Contributions to Born's new theory of the electromagnetic field, Proc. Roy. Soc. (London) A 150 (1935) 465.

[25] E.A. Ivanov, B.M. Zupnik, Bispinor auxiliary fields in duality-invariant electrodynamics revisited: the $U(N)$ case, in preparation. 